\title{
Expression of brain-specific angiogenesis inhibitor-1 and association with p53, microvessel density and vascular endothelial growth factor in the tissue of human bladder transitional cell carcinoma
}

\author{
DAWEI TIAN, HAILONG HU, YAN SUN, YANG TANG, MINGDE LEI, LIWEI LIU, \\ RUIFA HAN and CHANGLI WU
}

\begin{abstract}
Department of Urology, The Second Hospital of Tianjin Medical University,
\end{abstract} Tianjin Institute of Urology, Tianjin 300211, P.R. China

Received May 2, 2014; Accepted February 17, 2015

DOI: $10.3892 / \mathrm{mmr} .2015 .3984$

\begin{abstract}
The aim of the present study was to investigate the expression levels of brain-specific angiogenesis inhibitor-1 (BAI-1) in bladder transitional cell carcinoma (BTCC) at different stages and the mechanism by which it inhibits tumor endothelial cell proliferation. Normal bladder mucosa biopsy specimens were obtained as the control group, and human BTCC biopsy specimens were used as the study group. Immunohistochemical assays were used to detect the expression levels of BAI-1, vascular endothelial growth factor (VEGF) and mutant p53, in addition to microvessel density (MVD) in the tissues. Western blotting was used to analyze the differential expression of BAI-1 in the two samples. Statistical analysis was performed, which indicated that BAI-1 expression levels in the normal bladder mucosa group were significantly higher than those in the BTCC group and were associated with clinical staging. BAI-1 levels in the T1 stage BTCC tissues were higher than those in the T2-4 stage BTCC tissues $(\mathrm{P}<0.05)$. BAI-1 expression levels were negatively correlated with those of VEGF $(r=-0.661, \mathrm{P}<0.001)$, mutant p53 ( $\mathrm{r}=-0.406, \mathrm{P}=0.002)$ and with the MVD ( $\mathrm{r}=-0.675$, $\mathrm{P}<0.001)$. BAI-1 may be involved in the negative regulation of BTCC microvascular proliferation, and its expression may be associated with a reduction in p53 mutations.
\end{abstract}

Correspondence to: Dr Changli Wu, Department of Urology, The Second Hospital of Tianjin Medical University, Tianjin Institute of Urology, 23 Ping Jiang Road, Hexi, Tianjin 300211, P.R. China E-mail: dwdoccn@126.com

Key words: brain-specific angiogenesis inhibitor-1, bladder epithelial cells, bladder urothelial carcinoma, vascular endothelium

\section{Introduction}

Bladder urothelial carcinoma is the most common urological malignancy, accounting for $3.2 \%$ of all neoplastic malignancies and is the fifth-most common cancer in males, according to 2008 cancer statistics (1). Of all malignancies of the male genitourinary system, $>90 \%$ of cases are urothelial carcinoma, which present the highest rates of morbidity and mortality. The recurrence rate is low following surgery without other treatments, and in $10-20 \%$ of cases, bladder transitional cell carcinoma (BTCC) eventually develops into muscle-invasive bladder cancer, for which the five-year survival rate is $<50 \%$ (2-6). Intravesical therapy has been demonstrated to be an effective treatment to reduce the recurrence and progression of bladder cancer, and intravesical administration of Bacillus Calmette-Guérin (BCG) vaccine is able to produce an improved therapeutic effect in high-risk, non-invasive bladder cancer (7).

Brain-specific angiogenesis inhibitor-1 (BAI-1) was initially described in 1997, and there have since been a number of studies on its expression in different types of cancer. Nam et al (8) examined 20 cases of brain tumor, 2 samples of normal brain tissue, 5 cases of benign brain tumor and 26 patients with malignant glioma, and identified that BAI-1 was expressed in all normal brain tissue, benign tumor tissues and 9 cases of malignant glioma, while no expression was observed in the other types of tumor. Their results indicated that the tumors that expressed BAI-1 were relatively sensitive to treatment, and had good prognoses, while patients lacking BAI-1 expression had poor prognoses. Yoshida et al (9) studied 62 cases of colorectal cancer and 40 samples of normal extraneoplastic colon mucosa, and observed that the levels of thrombospondin 2 and angiopoietin-2 expression were significantly higher, with reduced BAI-1 expression levels in the colorectal cancer samples compared with those in the control samples. Hatanaka et al (10) analyzed the BAI-1 gene expression in 48 cases of lung adenocarcinoma and immunohistochemically assessed the vascular density in the tumor samples using an anti-CD34 monoclonal antibody. 
BAI-1 gene expression was observed in 38/48 (79.2\%) cases of lung cancer. The density of tumor blood vessels in the BAI-1-positive tumors $\left(19.3 \pm 4.4 / \mu \mathrm{m}^{2}, 1.7 \pm 0.6 \%\right)$ was significantly reduced compared with that in the BAI-1-negative tumors $\left(75.5 \pm 42.7 / \mu \mathrm{m}^{2}, 5.5 \pm 1.5 \%\right)$, indicating that BAI-1 may serve a function in the inhibition of interstitial vascularization in lung adenocarcinoma. Thus, it was hypothesized that BAI-1 may be useful as an indicator of prognosis in urinary disease. It was therefore theorized in the present study that BAI-1 may also act as an indicator of prognosis in bladder urothelial carcinoma, and that BAI-1 is a potential novel target for the treatment of these tumors. The expression of BAI-1-associated proteins in bladder urothelial carcinoma was examined in the present study, in order to provide a theoretical basis for the application of BAI-1 in the treatment of bladder cancer.

\section{Patients and methods}

Patients. Tissue blocks from 131 patients with BTCC, pathologically confirmed between 2009 and 2011 in the Department of Urology, Second Hospital of Tianjin Medical University (Tianjin, China), were randomly selected, and all cases were diagnosed by experienced pathologists. Staging of bladder cancer was performed using the tumor, nodes and metastasis (TNM) classification of malignant tumors (UICC) (11). The mean age of participants was 64, with a range of 27-89 years; 88 cases were male and 43 cases were female. A total of 57 cases $(43.5 \%)$ were TNM stage T1, 50 cases $(38.2 \%)$ were T2, and 24 cases $(18.3 \%)$ were T3-4. In terms of grading, a total of 90 cases $(68.7 \%)$ were classified as stages G1-2 (well/moderately differentiated) and 41 cases (31.3\%) were stage G3 (poorly differentiated). In addition, 28 cases with normal bladder mucosa tissues were selected as controls. The study was approved by the ethics committee of the Second Hospital of Tianjin Medical University. Written informed consent was obtained from the patients.

Specimens. Fresh clinical BTCC specimens from 21 cases were resected and divided into two groups according to UICC TNM staging: 8 Patients were staged as T1 and 13 patients presented with BTCC at the advanced stages of T2-4. Normal bladder epithelial tissues from 9 cases were obtained by suprapubic prostatic hyperplasia enucleation. The concentration of the protein extracted from the tissue samples, which were frozen in liquid nitrogen, was detected, and the BAI-1 protein content in the tissues was measured.

Immunohistochemistry $(I H C)$. The following primary antibodies were purchased from Abcam (Cambridge, UK): Monoclonal mouse anti-human mutant p53 antibody (cat. no. ab179477; Abcam), polyclonal rabbit anti-human BAI-1 (cat. no. ab135907; Abcam), polyclonal rabbit anti-human CD34 antibody (cat. no. ab150060, Abcam) and polyclonal rabbit anti-human vascular endothelial growth factor (VEGF) antibody (cat. no. ab46154; Abcam). Antibodies were diluted at a 1:100 ratio. PBS was used for the negative control group, and bladder cancer tissue known to be positive was used as a positive control. Polink-2 plus ${ }^{\circledR}$ polymer HRP detection system for rabbit primary antibodies (two steps) and a 3,3'-diaminobenzidine kit were purchased from Beijing
Zhongshan Golden Bridge Biotechnology, Co. Ltd. (Beijing, China) and were used according to the manufacturer's instructions. Tumor cells in which the cytoplasm, nucleus or membrane appeared brown were considered positive. Six high-magnification fields of each slice were randomly selected (magnification, $\mathrm{x} 400$ ), and the average percentages of positive cells were calculated. The following scoring method was used: $25 \%, 0$ points; $26-50 \%, 1$ point; $51-75 \%$, 2 points; and $>75 \%, 3$ points. Staining intensity was categorized as follows: Uncolored, 0 points; pale yellow, 1 point; yellow or brown, 2 points; and brown, 3 points. According to the final points, 0-1 was (-), 2-3 points was weakly positive $(+), 4-5$ points was positive $(++)$ and $>5$ was strongly positive $(+++)$. Specimens which scored $(+)$ to $(+++)$ were considered positive. The microvessel density (MVD) counting method was as follows: According to the Tanigawa criterion (12), the high-MVD areas were filtered out initially under low magnification $(10 \times 10)$, then the microvascular number of the five horizons under the $\mathrm{x} 400$ field was selected and the average was considered to be the number of tumor microvessels. Ambiguously stained cells were not included in the results and single brown endothelial cells or cell colonies were included in the vessel count, while the blood vessels with thicker vasculature and the lumen area with a red cell number $>8$ were not counted. The Olympus BX51 Microscope system (Olympus Corporation, Tokyo, Japan) was used to obtain images of the IHC staining (magnification, x400. Six horizons of each slide were blindly and randomly selected and their images were captured, which were quantitatively analyzed with Image-Pro Plus image analysis software, version 4.1 (Media Cybernetics, Inc., Rockville, MD, USA). The total optical density (OD) value of the brown staining on each slide in each field was analyzed and calculated, and the average OD value of each field area was calculated prior to statistical analysis.

Western blot analysis. A total of $100 \mathrm{mg}$ of the respective tissue block was placed in a mortar and cut into pieces with clean scissors. A total of $400 \mu 1$ RIPA lysis buffer system (Santa Cruz Biotechnology, Inc., Dallas, TX, USA) was added to the mortar prior to grinding the tissue, which was then placed on ice. The process of grinding the tissue and placing it on ice was repeated several times to fully homogenize it. The protein concentration was quantified with a Dc protein assay reagent (Bio-Rad, Hercules, CA, USA). Equal quantities of protein were loaded and separated by $10 \%$ SDS-PAGE (for HAMP, 18\%) and then transferred onto polyvinylidene difluoride membranes (Bio-Rad). The membranes were incubated overnight with the appropriate primary antibodies. Bound antibodies were then visualized using alkaline phosphatase-conjugated secondary antibodies. Quantitative analysis was performed using NIH Image $\mathrm{J}$ version $1.32 \mathrm{j}$ software (National Institutes of Health, Bethesda, MD, USA).

Statistical analysis. The $\chi^{2}$ test and Spearman's correlation analysis were used to analyze the associations between the clinicopathological parameters and IHC results. All data were analyzed using SPSS software, version 17.0 (SPSS, Inc., Chicago, IL, USA). $\mathrm{P} \leq 0.05$ was considered to indicate a statistically significant difference. 
Table I. Expression of BAI-1 in bladder epithelial cells.

\begin{tabular}{|c|c|c|c|c|c|c|c|}
\hline \multirow[b]{2}{*}{ Group } & \multicolumn{4}{|c|}{ BAI-1 immunohistochemistry } & \multirow[b]{2}{*}{ Total (\%) } & \multirow[b]{2}{*}{$\chi^{2}$ value } & \multirow[b]{2}{*}{ P-value } \\
\hline & $-(\%)$ & $+(\%)$ & $++(\%)$ & $+++(\%)$ & & & \\
\hline BHP & $0(0.00)$ & $0(0.00)$ & $8(30.1)$ & $20(69.9)$ & $28(100)$ & 108.738 & $<0.001$ \\
\hline BTCC & $86(65.6)$ & $32(24.4)$ & $11(8.4)$ & $2(1.5)$ & $131(100)$ & & \\
\hline
\end{tabular}

BAI-1, brain-specific angiogenesis inhibitor-1; BHP, normal bladder mucosa; BTCC, bladder transitional cell carcinoma.

Table II. Expression of p53, MVD and VEGF in bladder epithelial cells.

\begin{tabular}{|c|c|c|c|}
\hline Parameter & BHP $(n=28)$ & BTCC $(n=131)$ & P-value \\
\hline VEGF, n (\%) & & & $<0.001$ \\
\hline- & $27(96.5)$ & $34(26.0)$ & \\
\hline+ & $1(3.5)$ & $97(74.0)$ & \\
\hline p53, n (\%) & & & $<0.001$ \\
\hline- & $23(82.5)$ & $42(32.1)$ & \\
\hline+ & $5(17.5)$ & $89(67.9)$ & \\
\hline MVD (n/HPF) & $1.20 \pm 0.35^{\mathrm{a}}$ & $8.94 \pm 2.08$ & $<0.05$ \\
\hline
\end{tabular}

${ }^{a}$ Expressed as the mean \pm standard deviation. Mean data were obtained from 3 repetitions. $\mathrm{P}<0.05$ for MVD compared with BHP and BTCC. MVD, microvessel density; VEGF, vascular endothelial growth factor; BHP, normal bladder mucosa; BTCC, bladder transitional cell carcinoma; HPF, high power field.

Table III. Expression levels of p53, BAI-1 and VEGF in bladder epithelial cells $(n=159)$.

\begin{tabular}{lcccc}
\hline Type of tissue & $\mathrm{n}$ & BAI-1 (OD) & VEGF (OD) & p53 (OD) \\
\hline Normal & 28 & $176.81 \pm 97.01$ & $6.54 \pm 5.42$ & $23.8631 \pm 19.58$ \\
T1 & 57 & $69.28 \pm 79.62$ & $43.754 \pm 23.19$ & $116.29 \pm 34.92$ \\
T2-4 & 74 & $27.27 \pm 27.71$ & $217.28 \pm 124.99$ & $449.89 \pm 240.56$ \\
F-value & & 11.885 & 28.557 & 15.712 \\
P-value & & $<0.001$ & $<0.001$ & $<0.001$ \\
\hline
\end{tabular}

Expression levels are presented as the mean \pm standard deviation. Mean data were obtained from 3 repetitions. $\mathrm{P}<0.05$ represents the comparision between different groups for each index. OD values are presented as the mean \pm standard deviation. VEGF, vascular endothelial growth factor; BAI-1, brain-specific angiogenesis inhibitor-1; OD, optical density; IOD, immunohistochemical OD; T, tumor stage.

\section{Results}

MVD and expression of BAI-1, mutant p53 and VEGF in BTCC tissues. IHC analysis showed that BAI-1 was expressed in the cytoplasm and cell membrane, and VEGF was present in the cytoplasm, and mutant p53 was expressed in the nucleus (Fig. 1). Among the 28 normal bladder mucosa specimens, 28 (100\%), 5 (17.5\%) and 1 (3.5\%) were positive for BAI-1, mutant p53 and VEGF expression, respectively (Tables I and II). Among the 131 BTCC tissue specimens, $89(67.9 \%), 45(34.4 \%)$ and $97(74.0 \%)$ were positive for mutant p53, BAI-1 and VEGF expression. There were significantly fewer BAI-1-positive specimens in the BTCC group than in the normal bladder mucosa group ( $\mathrm{P}<0.01$; Table I, Fig. 2). Mutant p53 and VEGF expression were more common in the BTCC group than those in the normal bladder mucosa group ( $\mathrm{P}<0.05$; Table II). The MVD in the 28 normal bladder mucosa samples was 0.6-1.6/high power field (HPF) with an average of $1.2 \pm 0.35$. The MVD of the 131 BTCC samples was 4.4-13.4/HPF, with an average of $8.94 \pm 2.08(\mathrm{P}<0.01$ compared with normal bladder mucosa) (Table II).

Image analysis software was used to measure the ODs of all the IHC sections, and the results demonstrated that the mean OD value of BAI-1 staining in normal bladder mucosa samples was significantly higher than that in the BTCC samples (Table III). The OD values of the specimens correlated with 

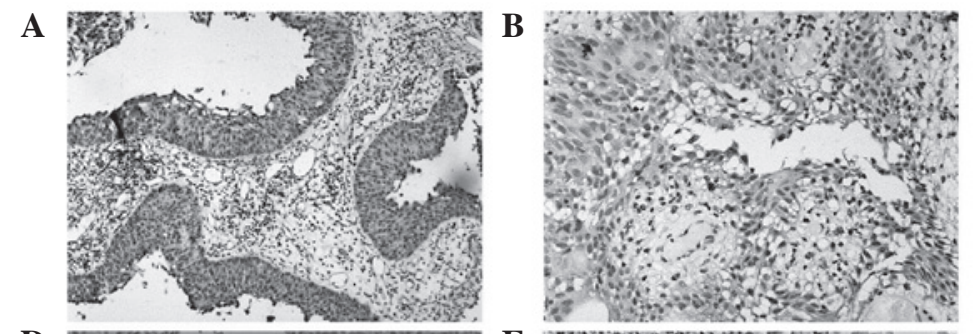

D

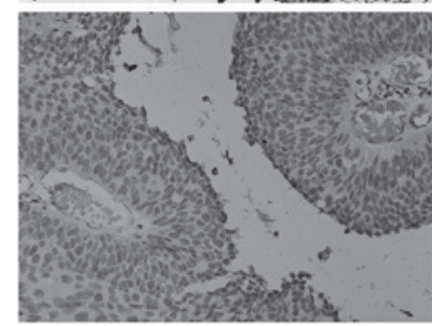

G

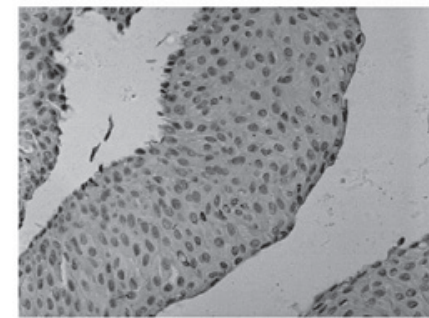

J

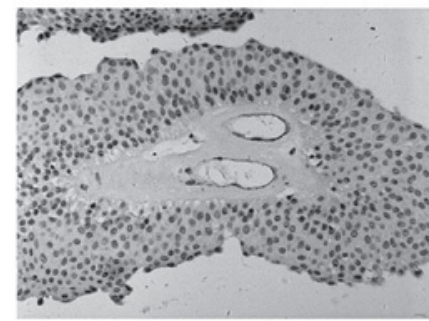

$\mathbf{E}$

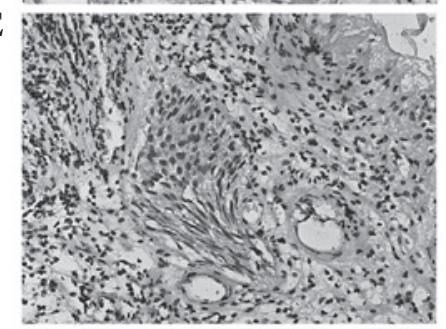

H

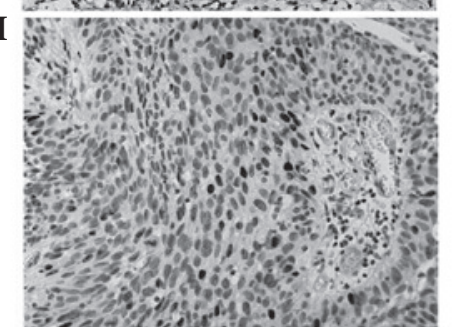

K

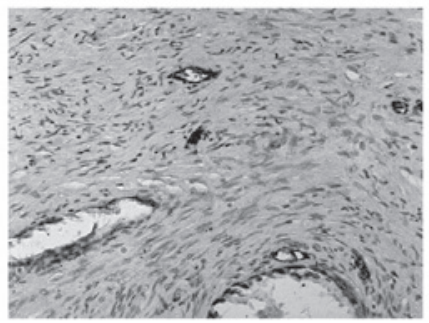

C

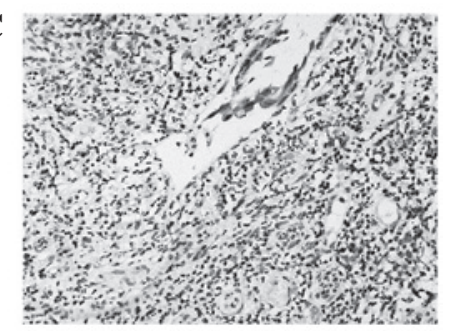

F

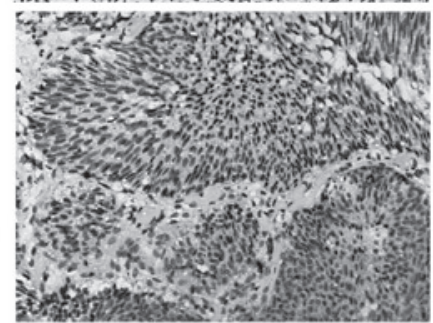

I

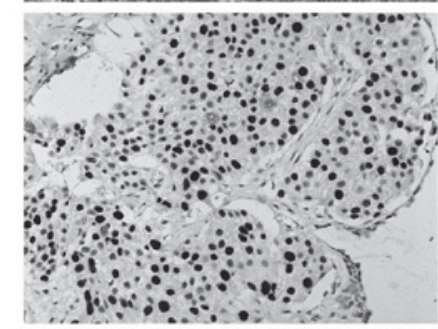

$\mathbf{L}$

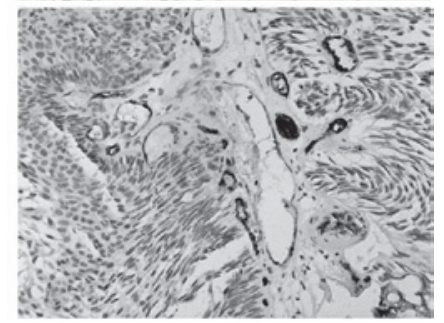

Figure 1. BAI-1, VEGF and $\mathrm{p} 53$ expression and MVD in bladder mucosa from the different groups. Representative images showing staining for BAI-1 in (A) normal bladder mucosa, (B) T1-stage BTCC and (C) T2-4-stage BTCC; VEGF in (D) normal bladder mucosa, (E) T1-stage BTCC and (F) T2-4-stage BTCC; p53 expression in (G) normal bladder mucosa, (H) T1-stage BTCC and (I) T2-4-stage BTCC; and MVD in (J) normal bladder mucosa, (K) T1-stage BTCC and (L) T2-4-stage BTCC (A-C, magnification x100; D-L, magnification x200). BAI-1, brain-specific angiogenesis inhibitor-1; VEGF, vascular endothelial growth factor; MVD, microvessel density; BTCC, bladder transitional cell carcinoma.

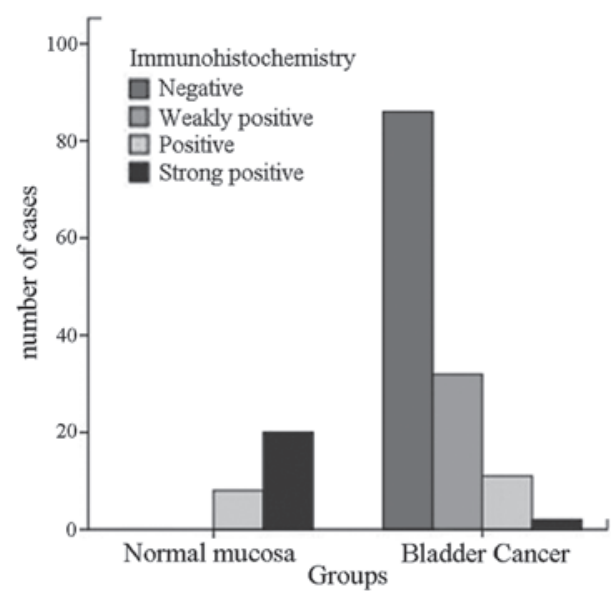

Figure 2. Brain-specific angiogenesis inhibitor-1 expression in normal bladder and urothelial cell carcinoma tissues.

the clinical stage, consistent with the statistics of positive IHC staining scores. The OD analysis results for mutant p53 were also consistent with those of VEGF (Table III, Fig. 3). As the statistical method to determine the MVD used the capillary number density as a measurement, the OD analysis method was not used to measure the MVD.

Association of BAI-1 with clinicopathological parameters of epithelial cancer. The association between BAI-1, VEGF, mutant p53 and MVD and age, gender, tumor grade, stage and number were further analyzed. Mutant p53 was observed to be positively correlated with the tumor grade, stage and whether multiple occurrence existed or not. With the increasing tumor grade and stage, the rate of positivity for mutant p53 expression increased correspondingly ( $\mathrm{P}<0.001$; Table IV), the rate in the multiple tumor group (93.2\%) was significantly higher than that in the single tumor group $(64.4 \% ; \mathrm{P}<0.001)$. The correlation between mutant $\mathrm{p} 53$ expression and age or gender was not identified to be statistically significant $(\mathrm{P}>0.05)$ (Table IV).

BAI-1 was negatively correlated with tumor grade, stage and multiple tumor occurrence (Table IV); with increasing tumor grade and stage, the BAI-1-positive rate decreased correspondingly $(\mathrm{P}<0.01)$ (Tables IV and V, Fig. 4). The BAI-1-positive rate in the multiple BTCC group (25\%) was significantly lower than that in the single group $(39.1 \% ; \mathrm{P}<0.05)$. No significant 
Table IV. Association between BAI-1, p53, VEGF and MVD and clinicopathological features in 131 cases of bladder cancer.

\begin{tabular}{|c|c|c|c|c|c|c|c|c|}
\hline \multirow[b]{2}{*}{ Characteristic } & \multirow[b]{2}{*}{ Total, n (\%) } & \multicolumn{2}{|c|}{$\mathrm{p} 53, \mathrm{n}(\%)$} & \multicolumn{2}{|c|}{ BAI-1, n (\%) } & \multicolumn{2}{|c|}{ VEGF, n (\%) } & \multirow{2}{*}{$\begin{array}{c}\mathrm{MVD}^{\mathrm{a}} \\
(\mathrm{n} / \mathrm{HPF})\end{array}$} \\
\hline & & - & + & - & + & - & + & \\
\hline Total (n) & & 42 & 89 & 86 & 45 & 34 & 97 & \\
\hline \multicolumn{9}{|l|}{ Age (years) } \\
\hline$\leq 64$ & $53(40.3)$ & $18(33.3)$ & $35(63.7)$ & $36(67.9)$ & $17(32.1)$ & $16(30.2)$ & $37(69.8)$ & $8.80 \pm 2.31$ \\
\hline$>64$ & $78(59.7)$ & $24(30.8)$ & $54(69.2)$ & $50(64.1)$ & $28(65.9)$ & $18(23.1)$ & $60(76.9)$ & $9.06 \pm 1.69$ \\
\hline P-value & & 0.142 & & 0.129 & & 0.334 & & 0.58 \\
\hline \multicolumn{9}{|l|}{ Gender } \\
\hline Male & $88(67.2)$ & $28(31.8)$ & $60(68.2)$ & $57(64.8)$ & $31(35.2)$ & $25(27.9)$ & $63(72.1)$ & $8.67 \pm 2.11$ \\
\hline Female & $43(32.8)$ & $14(32.5)$ & $29(67.5)$ & $29(67.4)$ & 14 (32.6) & $9(20.9)$ & $34(79.1)$ & $8.93 \pm 1.79$ \\
\hline P-value & & 0.205 & & 0.143 & & 0.927 & & 0.62 \\
\hline \multicolumn{9}{|l|}{ Stage } \\
\hline $\mathrm{T} 1$ & $57(46.8)$ & $30(52.6)$ & $27(47.4)$ & $19(33.3)$ & 38 (66.7) & $27(47.4)$ & $30(42.6)$ & $6.79 \pm 1.17$ \\
\hline $\mathrm{T} 2-4$ & $74(53.2)$ & $12(16.2)$ & $62(83.8)$ & $67(90.5)$ & $7(9.5)$ & $7(9.5)$ & $67(90.5)$ & $9.88 \pm 1.87$ \\
\hline P-value & & $<0.01$ & & $<0.01$ & & $<0.01$ & & $<0.01$ \\
\hline \multicolumn{9}{|l|}{ Grade } \\
\hline G1-2 & $90(68.7)$ & $35(31.5)$ & $55(68.5)$ & $46(51.1)$ & 44 (48.9) & $32(35.6)$ & $58(64.4)$ & $7.63 \pm 1.46$ \\
\hline G3 & $41(31.3)$ & $4(9.8)$ & $37(90.2)$ & $40(97.6)$ & $1(2.4)$ & $2(4.9)$ & $39(95.1)$ & $9.55 \pm 2.07$ \\
\hline P-value & & 0.01 & & $<0.01$ & & $<0.01$ & & $<0.01$ \\
\hline \multicolumn{9}{|l|}{ Multiplicity } \\
\hline Single & $87(66.4)$ & 39 (35.6) & $48(64.4)$ & $53(60.9)$ & $34(39.1)$ & $27(31.0)$ & $60(69.0)$ & $8.01 \pm 1.90$ \\
\hline Multiple & $44(25.0)$ & $3(6.8)$ & $41(93.2)$ & $33(75.0)$ & $11(25.0)$ & $7(15.9)$ & $37(84.1)$ & $9.47 \pm 2.31$ \\
\hline P-value & & $<0.01$ & & $<0.05$ & & $<0.05$ & & 0.17 \\
\hline
\end{tabular}

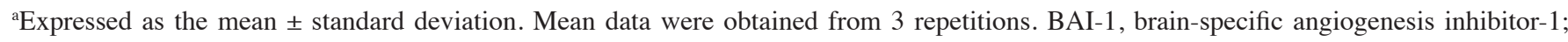
VEGF, vascular endothelial growth factor; MVD, microvessel density.

Table V. BAI-1 expression in tissues at different clinical stages of bladder transitional cell carcinoma.

\begin{tabular}{|c|c|c|c|c|c|c|c|}
\hline \multirow[b]{2}{*}{ Stage } & \multicolumn{4}{|c|}{ BAI-1 immunohistochemistry, n (\%) } & \multirow[b]{2}{*}{ Total, n (\%) } & \multirow[b]{2}{*}{$\chi^{2}$ value } & \multirow[b]{2}{*}{ P-value } \\
\hline & - & + & ++ & +++ & & & \\
\hline $\mathrm{T} 1$ & $27(47.4)$ & $20(35.1)$ & $8(14.0)$ & $2(3.5)$ & $57(100)$ & \multirow{3}{*}{17.138} & \multirow{3}{*}{$<0.001$} \\
\hline T2-4 & $59(79.7)$ & $12(16.2)$ & $3(4.1)$ & $0(0.0)$ & $74(100)$ & & \\
\hline Total & 86 & 32 & 11 & 2 & 131 & & \\
\hline
\end{tabular}

BAI-1, brain-specific angiogenesis inhibitor-1.

correlation was observed between BAI-1 expression and age or gender.

VEGF was positively correlated with tumor grade, stage and multiple tumor occurrence; as the tumor grade and stage increased, the rate of positivity for VEGF expression increased accordingly $(\mathrm{P}<0.01$; Table IV). The rate of positivity for VEGF expression in the multiple BTCC group (84.1\%) was significantly higher than that in the single BTCC group $(69.0 \%)(\mathrm{P}<0.05$; Table IV). The correlation of VEGF expression with age or gender was not statistically significant.
The MVD was positively correlated with the tumor grade and stage (Table IV). As the tumor grade and stage increased, the MVD in each high-magnification microscopic field increased correspondingly. The MVD in the multiple BTCC group was not significantly higher than that in the single BTCC group. Correlations between MVD and age, gender and multiple occurrence were not identified as significant (Table IV).

Correlation analysis. The IHC ODs were used to perform a correlation analysis between BAI-1 and mutant p53 and 

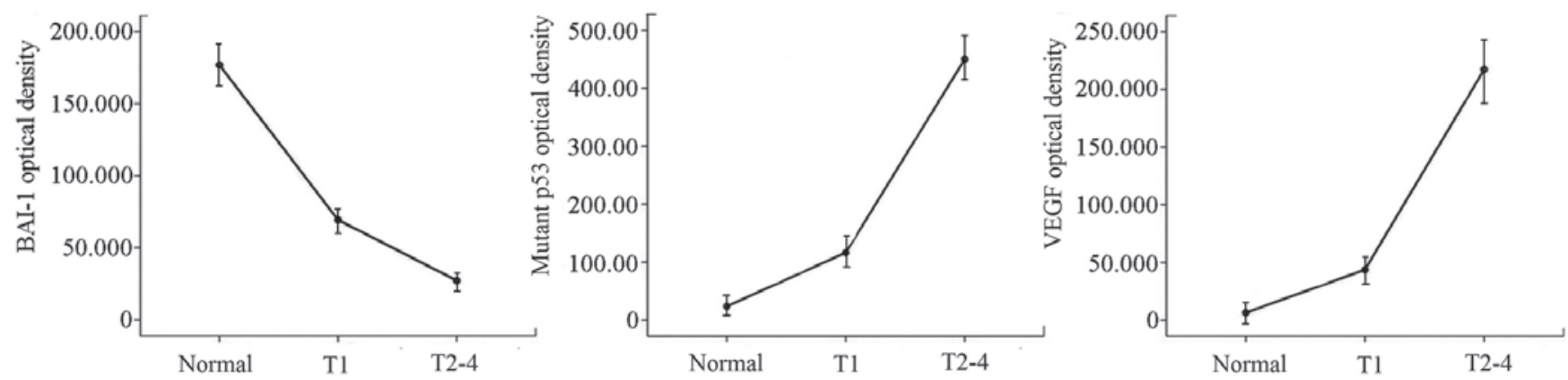

Figure 3. Mean optical density of BAI-1, p53 and vascular endothelial growth factor in different urothelial tissue specimens assessed by immunohistochemistry. BAI-1, brain-specific angiogenesis inhibitor-1. Values are presented as the mean \pm standard deviation.

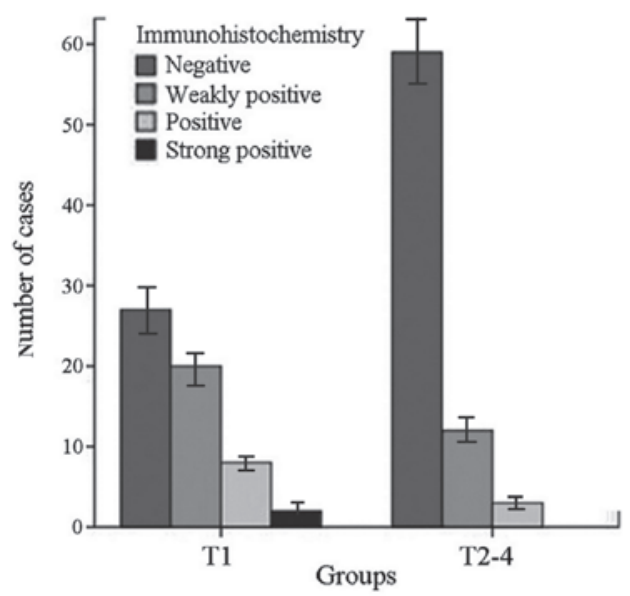

Figure 4. Brain-specific angiogenesis inhibitor-1 content in tissues at $\mathrm{T} 1$ and T2-4 clinical stages. Values are presented as the mean \pm standard deviation.

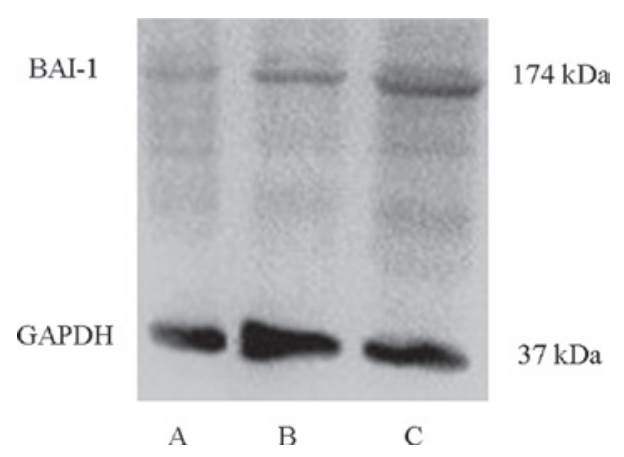

Figure 5. BAI-1 protein expression in bladder urothelial carcinoma at different stages. (A) T2-4; and (B) T1 bladder transitional cell carcinoma; and (C) normal bladder mucosa. BAI-1, brain-specific angiogenesis inhibitor-1.

VEGF (Table III). The results indicated a negative correlation with the average ODs, which were more accurate than the IHC scoring values; therefore, the mean ODs were used for the calculations. In the Spearman's rank correlation analysis, BAI-1 was identified to be negatively correlated with mutant p53 ( $\mathrm{r}=-0.675, \mathrm{P}<0.001), \mathrm{VEGF}(\mathrm{r}=-0.661, \mathrm{P}<0.001)$ and MVD $(\mathrm{r}=-0.406, \mathrm{P}=0.002)$, indicating that BAI-1 may be regulated by mutant $\mathrm{p} 53$, and this regulation is lost following the mutation of mutant $\mathrm{p} 53$, resulting in the reduction of BAI-1 expression. In addition, BAI-1 regulated the expression of VEGF; VEGF was upregulated and the capillary number

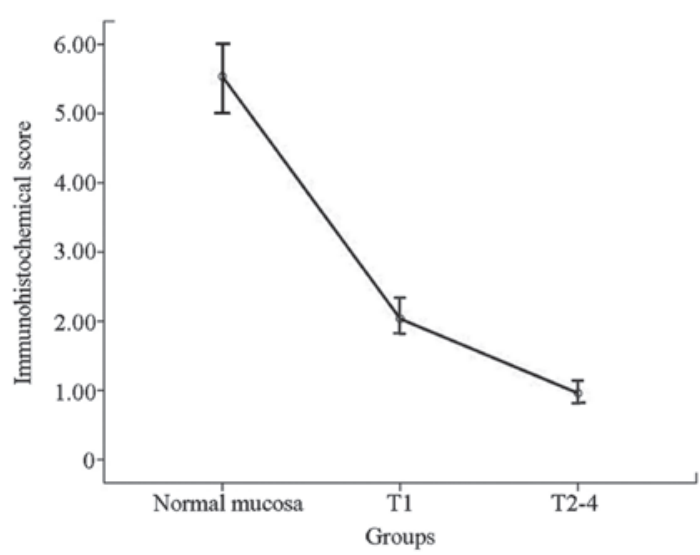

Figure 6. immunohistochemical score for brain-specific angiogenesis inhibitor-1 staning for samples at different clinical stages. Values are presented as the mean \pm standard deviation.

increased when a reduction in the levels of BAI-1 expression was observed.

BAI-1 decreases with the increase in tumor score. The results of the western blot analysis conducted on the freshly resected bladder cancer samples and normal bladder tissues were consistent with those of the IHC analysis. The BAI-1 protein content in the BTCC group was lower than that in the corresponding normal bladder mucosa, and was correlated with the clinical stage. In stage T1 samples, the BAI-1 content was significantly reduced as sompared with that in normal bladder mucosa, while BAI-1 was absent in T2-4 urothelial carcinoma tissues was absent (Fig. 5). In analogy with the western blot results, correlation of tumor staging with immunohistochemical scoring of BAI-1 also showed that BAI-1 was significantly decreased with increasing tumor stage (Fig. 6).

\section{Discussion}

In a study of functional p53 binding sites in human brain glioma in 1997, a novel target gene was isolated and termed BAI-1 (13). Although initially identified in glioma tissues, subsequent studies demonstrated that BAI-1 existed in other tissues, including kidney and colon, and was associated with the clinical tumor stage indicating the progression of the malignancy; the BAI-1 content was reduced or absent with the increase in tumor stage. Thus, the present study aimed to 
quantify the normal bladder mucosa paraffin tissue sections by IHC and detected fresh normal bladder tissue protein by western blot analysis. It was indicated that BAI-1 existed in normal bladder tissues. By analyzing 131 paraffin-embedded specimens from patients with BTCC and 21 specimens from patients with fresh urothelial carcinoma, it was demonstrated that BAI-1 protein levels in the bladder cancer samples were significantly lower than those in the normal bladder tissues; this change in protein levels exhibited a correlation with the clinical stage and whether multiple occurrence existed or not. BAI-1 levels were reduced with the increase of clinical stage; BAI-1 was almost absent in the T2-4 tumor tissues, and BAI-1 levels in multiple BTCC were significantly lower than those in the single tumors. The present study indicated that BAI-1 was expressed in a larger percentage of normal bladder epithelial tissues compared with that of urothelial cell carcinoma samples. Furthermore, the content of BAI-1 was negatively correlated with the staging of bladder carcinoma. BAI-1 may serve an important function in the suppression of tumor development.

BAI-1 is a p53 target gene, and the present study hypothesized that the reduced levels of BAI-1 in BTCC may be associated with a reduction of p53 mutations in tumor tissues. p53 is one of the most common established suppressor genes observed in the study of human malignancies. Previous studies have demonstrated that numerous oncogenes or tumor suppressor genes can recognize and bind to p53, thus regulating it at the transcriptional level (14). In the stage of tumor progression, p53 gene mutation leads to angiogenesis, which is conducive to the rapid growth of the tumor, and the tumor often enters the late tumor stage. The p53 gene can be divided into wild-type and mutant-type. The wild-type is an unstable regulatory protein with a half-life of $20 \mathrm{~min}$. The p53 protein most commonly detected in urothelial carcinoma by IHC methods is the mutant-type. The mutant p53 protein expression rate increases with the progression of the malignancy, reflecting that the high degree of expression of mutant p35 in bladder cancer causes its aggressiveness and high metastatic potential (15). Expression of mutant p53 protein in bladder cancer tissues has been indicated to be significantly correlated with grading and staging. The increase of mutant $\mathrm{p} 53$ protein was a result of the inactivation of wild-type p53, suggesting that the action of the p53 tumor suppressor gene was weakened or lost $(15,16)$. In the present study, the rate of mutant $\mathrm{p} 53$ expression in low-grade, low-stage and in single-tumor bladder cancer was lower than that in the high-grade, high-stage and multiple-tumor bladder cancer. The upregulation of mutant p53 expression levels in the present study was negatively correlated with the reduction of BAI-1 expression levels, indicating that the mutations and inactivation of p53 in bladder cancer may lead to BAI-1 downregulation.

As the tumor diameter reaches $>1-2 \mathrm{~mm}$, tumor tissues are challenged to obtain sufficient oxygen and nutrients, dependent on the growth of further blood vessels (17). The present study observed that BAI-1 expression was negatively correlated with VEGF and MVD. The BAI-1 extracellular fragment comprises five repeats of the TSP-1 fragment that was initially isolated from thrombin-stimulated platelet membrane by Baenziger et al (18). Their in vivo experiments demonstrated that TSP-1 was an endogenous angiogenesis inhibitor in the tumor microenvironment. Other studies have indicated that TSP-1 can inhibit tumor growth and the occurrence and development of tumors (19-21). TSP-1 and its receptor CD36 serve an important role in the inhibition of angiogenesis. The endothelial cells of small blood vessels and certain epithelial and stromal cells may express CD36. CD36 is required for TSP-1 binding and the inhibition of angiogenesis. In previous in vitro experiments, CD36 was able to mediate TSP-1 to inhibit the formation of endothelial cells and migration of blood vessels $(22,23)$. The outer membrane structure of BAI-1 has five TSP-1 repeats, which supports the theory of an anti-vascular function via the inhibition of endothelial cell proliferation (24).

VEGF is a cytokine of the platelet-derived growth factor family that is widely distributed in the brain, kidney, liver and other tissues in humans and other animals, and can produce numerous biological effects. VEGF is able to promote the proliferation of endothelial cells, increase vascular permeability, promote angiogenic support (25) and inhibit apoptosis of tumor cells. TSP-1 may affect the activity of VEGF through the following mechanisms: TSP-1 is able to inhibit the activity of matrix metalloproteinase-9 so that the extracellular matrix releases VEGF. This leads to tumor matrix formation, angiogenesis and tumor cell transfer. Furthermore, TSP-1 is able to combine with VEGF to mediate the uptake and clearance of VEGF (26).

In the 1990s, Folkman (27) demonstrated that tumor growth occurred in two distinct phases, e.g. the initial slow growth phase of blood vessels and the rapid growth phase, which confirms that the tumor blood vessels were newly generated vessels. New blood vessel network systems provide adequate oxygen and nutrients for tumor growth to ensure the rapid proliferation of tumor cells. Under normal circumstances, the process of angiogenesis is under strict control, while during tumorigenesis, a loss of cell cycle regulation and a switch from the suppression phenotype of capillaries into the active phenotype is present (28). MVD is used in tumor vasculature as the gold standard for evaluation of tumor-induced angiogenesis, and is closely associated with tumor malignant behavior (recurrence and metastasis) (29). Bergers and Benjamin (30) indicated that vascular tissue formation reflects the aggressiveness of the tumor, and a high MVD is associated with high probability of tumor cells entering the blood circulation; the tumor invasion and metastasis potential of malignant tumors increases as with increasing MVD. Therefore, MVD can be used to characterize the tumor growth, invasion ability, malignancy potential and prognosis to a certain extent.

In the present study, BAI-1 was negatively correlated with VEGF expression and MVD in the clinical samples. Based on the structure and function of BAI-1 binding fragments in the outer membrane, it was theorized that BAI-1 inhibits the formation of vascular endothelial cells and microvascular proliferation by inhibiting the expression of VEGF via TSP-1. Thus, low BAI-1 expression may promote tumor proliferation, invasion and metastasis.

BAI-1 is a G protein-coupled receptor (GPCR), which is a type of transmembrane receptor protein with seven transmembrane helices (31). GPCRs combine and regulate the activity of $\mathrm{G}$ proteins, which are a vital class of signaling receptor 
molecules (32). Diseases associated with the GPCRs are numerous, and $\sim 40 \%$ of modern drugs use GPCRs as therapy targets. The aim of research and development of modern drugs is to locate, identify and prepare targets of drug screening (molecular drug targets). Drug targets are drug binding sites, including the locui, receptors, enzymes, ion channels, nucleic acids and other biological macromolecules. Identification of novel drug targets is the initial task in drug development. As a member of the GPCR family, BAI-1 has the potential to become a novel target for anti-angiogenic therapy in the future.

In summary, BAI-1 expression was assessed in human bladder epithelial cells from patients with BTCC or in healthy bladder mucosa, and the levels of BAI-1 expression were demonstrated to be associated with the clinical stage as well as the multiplicity of tumor occurrence. The BAI-1 downregulation in urothelial carcinoma may be associated with p53 mutations. As a target protein of p53 regulation, BAI-1 is downregulated when wild-type p53 becomes mutated. The reduction or absence of BAI-1 may result in the increase in VEGF levels, leading to an increase in tumor microvessel density, thus prompting tumorigenesis and tumor progression. Therefore, BAI-1 protein levels have the potential to be a predictor of malignancy in tumor prognosis. Additionally, the study of the BAI-1 pathway in bladder urothelial carcinoma will contribute to the elucidation of the pathogenesis of bladder cancer and provide evidence for the use of inhibitors, with BAI-1 as a target. Thus, BAI-1 may be a novel target for cancer therapy.

\section{References}

1. Jemal A, Siegel R, Ward E, et al: Cancer statistics, 2008. CA Cancer J Clin 58: 71-96, 2008.

2. Rübben H, Lutzeyer W, Fischer N, Deutz F, Lagrange W and Giani G: Natural history and treatment of low and high risk superficial bladder tumors. J Urol 139: 283-285, 1988.

3. Sharifiaghdas F and Beigi FM: Impalpable testis: laparoscopy or inguinal canal exploration? Scand J Urol Nephrol 42: $154-157,2008$.

4. Reuter VE: The pathology of bladder cancer. Urology 67 (Suppl 1): 11-18, 2006.

5. Sylvester RJ, van der Meijden AP and Lamm DL: Intravesical bacillus Calmette-Guerin reduces the risk of progression in patients with superficial bladder cancer: a meta-analysis of the published results of randomized clinical trials. J Urol 168: 1964-1970, 2002.

6. Lamm DL, Blumenstein BA, Crissman JD, et al: Maintenance bacillus Calmette-Guerin immunotherapy for recurrent TA, T1 and carcinoma in situ transitional cell carcinoma of the bladder: a randomized Southwest Oncology Group Study. J Urol 163: $1124-1129,2000$.

7. Davis JW, Sheth SI, Doviak MJ and Schellhammer PF: Superficial bladder carcinoma treated with bacillus Calmette-Guerin: progression-free and disease specific survival with minimum 10-year followup. J Urol 167: 494-501, 2002.

8. Nam DH, Park K, Suh YL and Kim JH: Expression of VEGF and brain specific angiogenesis inhibitor-1 in glioblastoma: prognostic significance. Oncol Rep 11: 863-869, 2004.

9. Yoshida Y, Oshika Y, Fukushima Y, et al: Expression of angiostatic factors in colorectal cancer. Int J Oncol 15: 1221-1225, 1999

10. Hatanaka H, Oshika Y, Abe Y, et al: Vascularization is decreased in pulmonary adenocarcinoma expressing brain-specific angiogenesis inhibitor 1 (BAI1). Int J Mol Med 5: 181-183, 2000.

11. Sobin LH and Wittekind C: TNM Classification of Malignant Tumors (UICC). 6th edition. Wiley-Liss, New York, NY, pp199-203, 2002.
12. Kagan VE, Kisin ER, Kawai K, et al: Toward mechanism-based antioxidant interventions: lessons from natural antioxidants. Ann NY Acad Sci 959: 188-198, 2002.

13. Kuwano M, Nakamura Y and Tokino T: A novel brain-specific p53-target gene, BAI1, containing thrombospondin type-1 repeats inhibits experimental angiogenesis. Oncogene 15: 2145-2150, 1997.

14. Kim E and Deppert W: The versatile interactions of p53 with DNA: when flexibility serves specificity. Cell Death Differ 13: $885-889,2006$.

15. Piaton E, Faÿnel J, Ruffion A, Lopez JG, Perrin P and Devonec M: p53 immunodetection of liquid based processed urinary samples helps to identify bladder tumours with a higher risk of progression. Br J Cancer 93: 242-247, 2005.

16. Wei M, Wanibuchi H, Morimura K, et al: Carcinogenicity of dimethylarsinic acid in male F334 rats and genetic alterations in induced urinary bladder tumors. Carcinogenesis 23: 1387-1397, 2002.

17. Anderson JC, McFarland BC and Gladson CL: New molecular targets in angiogenic vessels of glioblastoma tumours. Expert Rev Mol Med 10: e23, 2008.

18. Baenziger NL, Brodie GN and Majerus PW: A thrombin-sensitive protein of human platelet membranes. Proc Natl Acad Sci USA 68: 240-243, 1971.

19. Naumov GN, Bender E, Zurakowski D, et al: A model of human tumor dormancy: an angiogenic switch from the nonangiogenic phenotype. J Natl Cancer Inst 98: 316-325, 2006.

20. Okada K, Hirabayashi K, Imaizumi T, et al: Stromal thrombospondin-1 expression is a prognostic indicator and a new marker of invasiveness in intraductal papillary-mucinous neoplasm of the pancreas. Biomed Res 31: 13-19, 2010.

21. Greenaway J, Lawler J, Moorehead R, Bornstein P, Lamarre J and Petrik J: Thrombospondin-1 inhibits VEGF levels in the ovary directly by binding and internalization via the low density lipoprotein receptor-related protein-1 (LRP-1). J Cell Physiol 210: 807-818, 2007.

22. Dawson DW, Pearce SF, Zhong R, Silverstein RL, Frazier WA and Bouck NP: CD36 mediates the in vitro inhibitory effects of thrombospondin-1 on endothelial cells. J Cell Biol 138: 707-717, 1997.

23. Dawson DW, Volpert OV, Pearce SF, et al: Three distinct D-amino acid substitutions confer potent antiangiogenic activity on an inactive peptide derived from a thrombospondin-1 type 1 repeat. Mol Pharmacol 55: 332-338, 1999.

24. Koh JT, Kook H, Kee HJ, et al: Extracellular fragment of brainspecific angiogenesis inhibitor 1 suppresses endothelial cell proliferation by blocking alphavbeta5 integrin. Exp Cell Res 294: 172-184, 2004.

25. Nicosia RF: What is the role of vascular endothelial growth factor-related molecules in tumor angiogenesis? Am J Pathol 153: $11-16,1998$

26. Kang SY and Watnick RS: Regulation of tumor dormancy as a function of tumor-mediated paracrine regulation of stromal Tsp-1 and VEGF expression. APMIS 116: 638-647, 2008.

27. Folkman J: What is the evidence that tumors are angiogenesis dependent? J Natl Cancer Inst 82: 4-6, 1990.

28. Elston CW and Ellis IO: Pathological prognostic factors in breast cancer. I. The value of histological grade in breast cancer: Experience from a large study with long-term follow-up. Histopathology 41: 154-161, 2002.

29. Kato T, Steers G, Campo L, et al: Prognostic significance of microvessel density and other variables in Japanese and British patients with primary invasive breast cancer. Br J Cancer 97: 1277-1286, 2007.

30. Bergers $\mathrm{G}$ and Benjamin LE: Tumorigenesis and the angiogenic switch. Nat Rev Cancer 3: 401-410, 2003.

31. El Moustaine D, Granier S, Doumazane E, et al: Distinct roles of metabotropic glutamate receptor dimerization in agonist activation and G-protein coupling. Proc Natl Acad Sci USA 109: 16342-16347, 2012.

32. New D C and Wong YH: Molecular mechanisms mediating the $\mathrm{G}$ protein-coupled receptor regulation of cell cycle progression. J Mol Signal 2: 2, 2007. 